\title{
An approach to hearing loss in children
}

\section{GJ Copley MBChB (UCT), FCS (ORL) SA \& NB Friderichs}

To cite this article: GJ Copley MBChB (UCT), FCS (ORL) SA \& NB Friderichs (2010) An approach to hearing loss in children, South African Family Practice, 52:1, 34-39, DOI: 10.1080/20786204.2010.10873928

To link to this article: http://dx.doi.org/10.1080/20786204.2010.10873928

(2) 2010 SAAFP. Published by Medpharm.

Published online: 15 Aug 2014.

Submit your article to this journal $\pi$

Џ Article views: 429

Q View related articles $\sqsubset$ 


\title{
An approach to hearing loss in children
}

\author{
${ }^{a}$ Copley GJ, MBChB (UCT), FCS (ORL) SA ${ }^{\text {b }}$ Friderichs NB, Communication Pathology (UP) \\ a University of Cape Town, Groote Schuur and Red Cross Children's hospitals, Cape Town, South Africa \\ b The Carel du Toit Centre, Ivan Tom's Infant Hearing Screening Programme, South Africa \\ Correspondence to: Dr Graeme Copley, e-mail: gjcopley@yahoo.com
}

Keywords: hearing loss in children; newborn hearing screening; Early Hearing Detection and Intervention (EHDI); otitis media

\begin{abstract}
Hearing loss in children is common and can be difficult to identify, especially in young children. There are a variety of pathologies involved in these children, which make the problem challenging to diagnose and manage. This update describes how new technologies have evolved over the last 10 years to assist in the early detection of hearing loss even in very young children. The article aims to highlight the role and assist the general practitioner in managing these children, so that effective intervention and rehabilitation can occur and the long-term benefit, namely that the children become functioning members of the hearing world, is achieved. This article is not a comprehensive review on the subject, but aims to remind the reader of risk factors and gives guidelines for the clinical assessment of a child with hearing loss. In addition, middle ear disease is also widespread and impacts significantly on the quality of life in this age group, and therefore these problems are also discussed for the sake of completeness. The Ivan Tom's infant hearing screening programme based in Cape Town is presented as a pilot project for future universal neonatal screening projects in South Africa.
\end{abstract}

(P) Peer reviewed. (Submitted: 2009-05-15, Accepted: 2009-07-27). ๑ SAAFP

SA Fam Pract 2010;52(1): 34-39

\section{Introduction}

The main aim of this article is to raise awareness among primary care physicians of the importance of identifying children with hearing loss as early as possible and referring them for appropriate intervention. It will hopefully also demonstrate the primary care practitioner's key role in the overall care of these children. There are many issues involved when a child has hearing loss, ranging from initially suspecting the problem, making the diagnosis, breaking the bad news the need for family support and counselling to initiating intervention and management and, very importantly, the follow-up, tracking, and monitoring of these children. The family physician should act as a crucial coordinator, liaison officer and director of proceedings, and also provide support to the child and family.

The child suffering from hearing loss frequently has other medical problems, be they genetic, cardiac, ophthalmologic, craniofacial, orthopaedic, etc. They will need to be assessed by paediatricians, audiologists, ophthalmologists, speech and language therapists and otorhinolaryngologists. The child may need a number of special investigations to be performed, to be fitted with hearing aids, surgery or just regular cleaning of an ear discharge and treatment for recurrent infections. The social impact on the family having a child with hearing loss is huge, and the ability of a primary health care physician to mobilise community support, refer to social workers for care grants and provide counselling for the child and family should never be underestimated.

In the modern competitive world in which we now live, having a healthy, well-educated population is of prime importance for a developing nation such as South Africa. The ability of our children to learn, become educated, communicate well and develop skills so that in the future they may become meaningful contributors to our society should be a priority for our health, education and social services. As stated in the 2006 World Development Report," "evidence supports the view that investing in early childhood has large impacts on children's health and readiness to learn and can bring important economic returns later in life - often greater than investments in formal education and training". The early identification and appropriate management of hearing loss in children can go a long way in achieving these goals.

\section{Prevalence of hearing loss}

Permanent disabling hearing impairment $(>40 \mathrm{~dB}$ hearing loss) is a significant contributor to the global burden 
of disease on individuals, families, communities and countries, affecting about 250 million people worldwide, as estimated in 2005. ${ }^{2}$ Two-thirds of these people live in developing countries and one in every four are of early childhood onset. ${ }^{2}$ Hearing impairment has a significantly higher prevalence than any other birth defect $^{3}$ and infant hearing loss is the most common congenital sensory birth defect with a prevalence of 4 to 6 per 1000 live births in a developing country like South Africa. ${ }^{4}$ This is higher than the usually quoted figure of 1 to 3 per 1000 births for developed countries. Preliminary reports in South Africa propose that approximately 17 babies are born with or will develop hearing loss in South Africa every day, $85 \%$ of these being in the public health sector. ${ }^{5}$ Risk factors, as listed below, are common to South Africa and increase the risk for infant hearing loss.

- Hearing loss in children is referred to as an epidemic because of its high prevalence.

- Hearing loss is commoner than Down's syndrome, cleft lip and palate and spina bifida.

- Hearing loss is referred to as the silent, overlooked epidemic of developing countries because of its invisible nature that prevents detection through routine clinical procedures. $^{6}$

\section{The impact of hearing loss in children}

Of all the sensory disabilities in early childhood, permanent hearing loss is of special interest because of its adverse effects on speech, language, cognitive and psychosocial development, and the subsequent impact on educational and vocational attainment. In landmark research by Yoshinaga-Itano et al, they showed that children diagnosed late with hearing loss had significantly poorer reading comprehension and language skills than those diagnosed before six months of age. ${ }^{7}$

Fortunately, there is something we can do about this. Further research evidence indicates that an infant with hearing loss with no other associated disabilities who receives early intervention within the first six months of life is likely to have linguistic, speech and cognitive development comparable to normal hearing peers by the age of two years in contrast to persistent delays for those who are identified late. $8,9,10$

A society is furthermore also severely burdened by hearing loss due to the extensive economic costs associated with it. Hearing loss without adequate intervention affects an individual's ability to obtain, perform in and keep a job, and it causes people to be isolated and stigmatised during the entire course of their lives. ${ }^{8,9}$ The income of individuals with hearing loss is reported to be 40 to $45 \%$ less than the hearing population in developed countries and will be even more pronounced in developing countries like South Africa, rendering those with hearing loss the poorest of the poor. ${ }^{11}$ In the United States, where this information is available, it has been estimated that the combined expense of specialised education and loss of productivity, results in an average lifetime cost for the government of more than US\$1 million. ${ }^{12}$

\section{Aetiology}

It is perhaps most practical to divide children with hearing loss into two broad groups: the newborns with a congenital or late-onset hereditary hearing loss, and the slightly older children with middle ear disease. The hearing loss associated with newborns is usually sensorineural in nature; it may be due to a genetic defect or acquired from the environment, for example a toxin exposure or an intrauterine infection. Then there are the older children, approximately two to six years of age, with mild to moderate hearing loss due to middle ear disease. Most frequently this is due to middle ear infection that has become chronic in nature, either a persistent middle ear effusion or a chronic perforation with or without an active infection.

The environmental causes of hearing loss, commonly associated with infectious diseases, such as pneumococcal and haemophilus respiratory infections, and epidemics such as HIV and TB, all account for substantial infant morbidity and mortality in developing countries. This is probably the reason for the higher prevalence of hearing loss in children in South Africa. Primary prevention through immunisation, health education and improved maternal and child health services is useful for addressing these environmental causes and will go a long way in reducing the burden of disease. However, it must be remembered that these interventions are limited in dealing with the full spectrum of neonatal hearing loss attributable to genetic or hereditary aetiologies.

\section{Hearing loss in newborns}

About $50 \%$ of hearing loss in this group is due to genetic factors. ${ }^{13}$ The embryological development of the ear is complex and vulnerable to genetic mishaps. About $15 \%$ of the genetically induced hearing losses have been described as a known genetic syndrome, for example Pendred's, Waardenberg's, Treacher Collin's, Alport's and Usher's syndromes. For the average primary care giver the chance of seeing one of these rare syndromes is quite remote. The other $35 \%$ of hereditary hearing loss have an isolated genetic deafness referred to as non-syndromic hereditary hearing loss. They are most frequently due to recessive mutations, at certain gene loci, which can, for example, affect plasma membrane proteins such as connexin 26 . 
This cell wall protein is involved with potassium transport, a crucial electrolyte in the function of the membranes and fluids of the inner ear. ${ }^{14}$ With modern biotechnology techniques, more and more of these abnormal genes are being identified, and their inheritance patterns are becoming better understood. It can therefore be useful for families with a history of congenital deafness to undergo genetic counselling and possibly testing, so that they may

\section{Table I: Risk indicators for infants and children} in South Africa ${ }^{16}$

\section{RISK-BASED SCREENING IN SOUTH AFRICA}

(Birth to 28 days of age)

An illness or condition requiring admission of 48 hours or greater to a neonatal intensive care unit

Stigmata or other findings associated with a syndrome known to include a sensorineural and/or conductive hearing loss

Family history of permanent childhood sensorineural hearing loss

Craniofacial anomalies, including those with morphological abnormalities of the pinna and ear canal

In-utero infection such as cytomegalovirus, herpes, toxoplasmosis rubella,

HIV or malaria

\section{RISK-BASED SURVEILLANCE IN SOUTH AFRICA}

\section{(Age 29 days to 2 years)}

Parental or caregiver concern regarding hearing, speech or language and/or

developmental delay

Family history of permanent childhood hearing loss

Stigmata or other findings associated with a syndrome known to include a sensorineural or conductive hearing loss or Eustachian tube dysfunction

Postnatal infections associated with sensorineural hearing loss, including bacterial meningitis

In-utero infections such as cytomegalovirus, herpes, rubella, syphilis, toxoplasmosis, HIV or malaria

Neonatal indicators - specifically hyperbilirubinaemia at a serum level requiring exchange transfusion, persistent pulmonary hypertension of the newborn associated with mechanical ventilation and conditions requiring the use of extracorporeal membrane oxygenation

Syndromes associated with progressive hearing loss, such as neurofibromatosis,

osteopetrosis and Usher's syndrome

Neurodegenerative disorders, such as Hunter's syndrome, or sensory motor

neuropathies, such as Friedreich's ataxia and Charcot-Marie-Tooth's syndrome

Head trauma

Recurrent or persistent otitis media with effusion for at least three months

HIV infection and malaria be aware of the risks for hearing loss in their progeny and make informed decisions about having children, and if they do have children, to be aware of the increased risk and monitor them closely. ${ }^{15}$

The other causes of congenital hearing loss are not hereditary, and are acquired during pregnancy or soon thereafter, or they arise from some sporadic unknown aetiology. These typically cause sensorineural hearing loss ranging from mild to profound in degree. ${ }^{18}$ Examples include the following:

- Intrauterine infections, including cytomegalovirus, HIV, toxoplasmosis, herpes simplex, syphilis, and rarely rubella (German measles)

- Complications associated with the Rhesus factor

- Prematurity and low birth weight babies

- Maternal diabetes

- Hypertension during pregnancy

- Hypoxic ischaemic encephalopathy

Following delivery there are well-known perinatal causes for hearing loss, such as the following:

- Hyperbilirubinaemia

- Drugs - especially those used in the neonatal ICU (aminoglycosides, frusemide)

- Neonatal meningitis

- Oxygen therapy

The Position Statement on Early Hearing Detection and Intervention (EHDI) programmes of the Health Professions Council of South Africa (HPCSA) has developed guidelines for assessing the risk for early hearing loss in children. ${ }^{14}$ Any infant or child with the risk factors shown in Table I should be red-flagged and assessed carefully for hearing loss as soon as possible.

\section{Hearing loss in older children}

As children mature, they undergo an immunological revelation; they move out of the protective confines of breast milk and motherly home care and are exposed to new foods, new environments, new viruses and bacteria. They develop frequent upper respiratory tract and gastrointestinal infections. An important part of the immune system for children exists as lymphoidal tissue in the nasopharynx and oropharynx: the adenoids and tonsils. These concentrations of lymphoid tissue, collectively referred to as Waldeyer's ring, survey all the intake of air, food and fluid, process antigens and manufacture immunoglobulins. They frequently become quite hypertrophied in children as they carry out this function. In addition to the short, flaccid Eustachian tube of childhood, the overall immune immaturity and the mechanical effect of enlarged adenoids and tonsils, children are susceptible to middle ear infections. ${ }^{17}$ The most 
common presentation is that of acute otitis media, which does not usually have any long-term effect on hearing. However, in a proportion of these children they may go on to develop complications, a chronic middle ear pathology, which will certainly have effects on their hearing. ${ }^{18}$

An as yet unpublished survey on the common causes for hearing loss in children was carried out at audiological clinics in Cape Town in 2007. Seventy children under the age

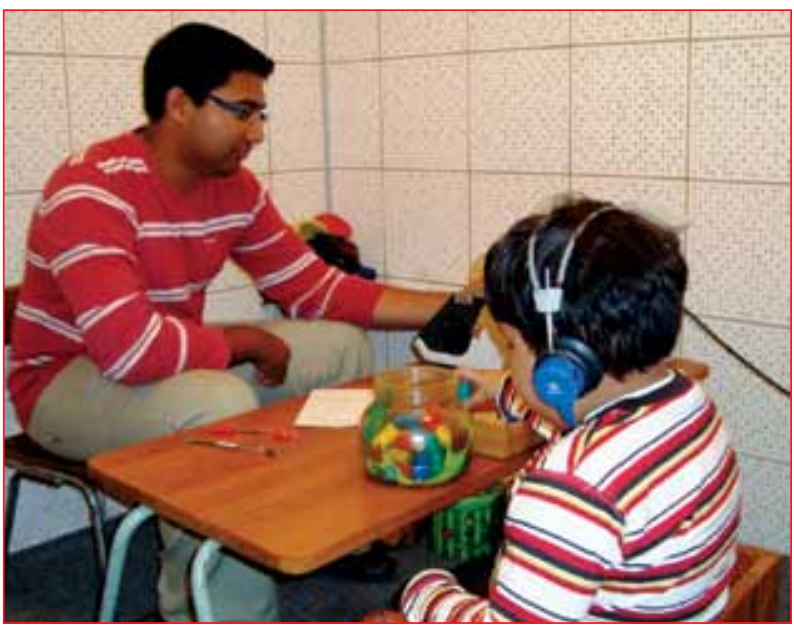

Figure 1: Play audiometry for testing older children

of ten years that had been diagnosed with hearing loss were included in the study. More than $50 \%$ of these children had hearing loss due to otitis media with effusion, also known as 'glue ear'. This occurs when the acute otitis media infection is resolved but a residue mucoid effusion is retained in the middle ear cleft and does not drain via the Eustachian tube. Usually this middle ear effusion creates a mild (20-30 $\mathrm{dB}$ ) conductive hearing loss and for the most part is selflimiting; the majority of children will get better without the need for any medical or surgical intervention. However, a portion of these children have a persistent effusion that lasts for more than three months. If this is the case then it is unlikely that the effusion will resolve spontaneously, and now with a prolonged mild hearing loss there may well be an effect on the speech and language development of the child as well as damage to the tympanic membrane and middle ear structures due to the chronic middle ear inflammation. ${ }^{19}$

The common acute otitis media can also cause an acute perforation of the tympanic membrane, and releases a mucopurulent discharge into the ear canal. Usually the infection resolves spontaneously or with the aid of antibiotic therapy, and the tympanic membrane then heals. But a proportion of these infected middle ears will go on to develop a chronic perforation, which can become colonised with bacteria and lead to an active chronic otitis media with concomitant hearing loss. This is usually quite easy to diagnose, as the child presents with a persistent or intermittent ear discharge. Careful cleaning of the ear canal with cotton wool mops, to clear out the discharge and debris and so to make way for the instillation of topical ear drops, is the most effective way to resolve the active infection. ${ }^{20}$ The tympanic membrane may spontaneously heal once the chronic infection is treated, but some children will have a persistent perforation, and may have other ossicular damage, which will lead to long-term hearing loss.

\section{The identification of hearing loss in children}

The variable pathologies involved and the range of age in presentation make this task a challenging one. It is simple enough in the older child who presents with a chronically discharging ear and complains of hearing loss, or it may have been noted at school by the teacher or picked up by a school nurse (see Figure 1). One would also imagine parents to be a good judge of the ability of a child to hear. This unfortunately does not seem to be the case. In research carried out at a hearing screening programme in Hong Kong, school children were assessed for otitis media with effusion. Prior to the screening the parents were questioned about any suspicions they may have that their child may be suffering from hearing loss. The sensitivity of parentsuspected hearing impairment to detect middle ear effusion was very low (19.7\%). In other words, if we were to rely on parental suspicion as the first screening, at least $80 \%$ of these cases, albeit mild cases of hearing loss, would have been missed. ${ }^{21}$

But still, a good history and examination are important in identifying hearing loss. A detailed family history to identify affected parents, siblings and relatives is imperative in the evaluation of the patient with hearing impairment. An evaluation of the neurological development and speech and language development can also assist. Failure to achieve the following speech and language milestones may indicate hearing loss and necessitate a hearing evaluation ${ }^{22}$ :

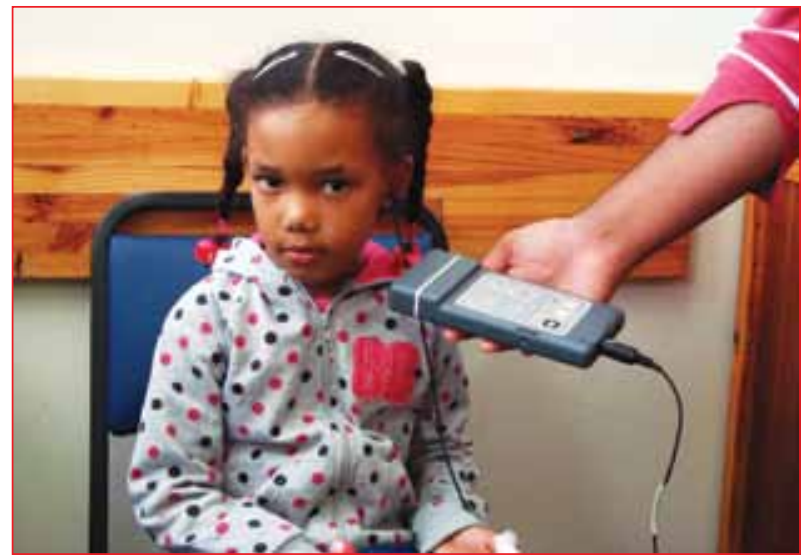

Figure 2: Objective testing using otoacoustic emissions 


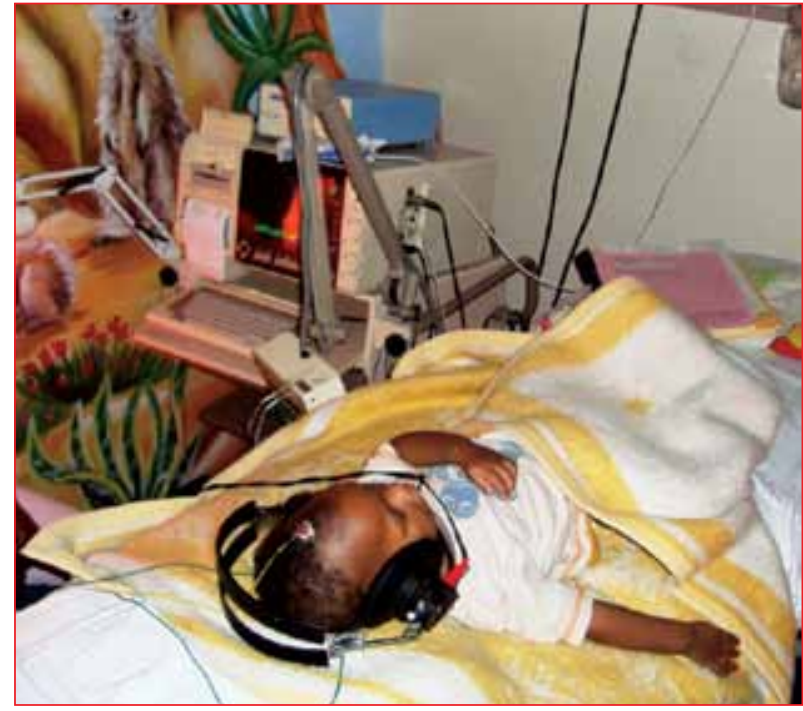

Figure 3: Objective testing using auditory brain stem response

- Birth to three months

- Startles to loud noise

- Awakens to sounds

- Blinks or widens eyes in response to noises

- Three to four months

- Quiets to mother's voice

- Stops playing, listens to new sounds

- Looks for source of new sounds that are not in sight

- Six to nine months

- Enjoys musical toys

- Coos and gurgles with inflection

- Says "mama"

- Twelve to fifteen months

- Responds to his or her name and the word "no"

- Follows simple requests

- Uses expressive vocabulary of three to five words

- Imitates some sounds

- Eighteen to twenty-four months

- Knows body parts

- Uses expressive vocabulary with two-word phrases (minimum of 20 to 50 words)

- $50 \%$ of speech is intelligible to strangers

- By 36 months

- Uses expressive vocabulary of four- to five-word sentences (approximately 500 words)

- $80 \%$ of speech is intelligible to strangers

- Understands some verbs

Because sensorineural hearing loss is associated with effects on virtually every organ, the physician must be familiar with the constellation of physical findings that may elucidate the aetiology of a patient's hearing impairment. Physical examination should include a complete evaluation of the ears, nose, throat, head and neck, along with an overall assessment of the child's general physical and neurologic status. Findings associated with hearing loss include microtia or atresia of the ear canal, cleft lip or palate, craniofacial abnormalities (e.g. micrognathia, facial asymmetry, microcephaly or craniosynostosis), cranial nerve weakness, heterochromia of the iris or other abnormalities of the ocular structures, vision impairment, goitre and skeletal abnormalities

\section{The role of universal screening of children for hearing loss}

Screening of newborns or infants before the age of three months has emerged within the last decade as an effective secondary prevention strategy for the early detection of disabling permanent hearing impairment. ${ }^{23}$ About 10 years ago, very little was known about this powerful intervention to resolve the difficulties in identifying young children with hearing loss. The development and availability of objective hearing tests using otoacoustic emissions (OAE) and/ or auditory brainstem response, which are suitable for testing babies from birth, have been a major impetus for this trend (see Figures 2 and 3). These screening tests are often automated, reliable, non-invasive and simple to use by non-specialists such as primary health care workers. Although universal or target screening of newborns is widely implemented in developed countries, its introduction in the developing world may be constrained by reservations concerning the necessity of such a programme because of prevailing adverse health and socio-economic conditions and restricted resources. ${ }^{5}$ However, the long-term implications of not initiating such programmes place an added economic burden on these developing countries.

At present the only 'formal' screening for hearing loss in children proposed by the South African Department of Health ${ }^{24}$ is the use of two hearing tests, namely the Voice test and the Swart Questionnaire for babies younger than 12 months. The primary health care worker asks the caregivers questions from the "Road to Health Card", namely if the child is able to "appear to listen" at three months and "turns to a loud noise" at six months, and conducts a voice test. With the introduction of the HPCSA Position Statement on EHDI programmes in South Africa, ${ }^{16}$ this is, however, slowly changing. Newborn hearing screening services in South Africa have been initiated in both the public and private sector. The private health care sector is mostly dependent on individual initiatives from private practice audiologists in hospitals, but is not mandated by hospital management and therefore remains mostly unstructured and unsystematic. ${ }^{5}$ Services in the public sector are far scarcer with no more than $7.5 \%$ of public hospitals providing some form of infant hearing screening. ${ }^{25}$ This poses dire consequences, 
considering that approximately $85 \%$ of the South African population rely on the public health system for health care and that $61 \%$ of children in South Africa live in poverty. ${ }^{5,26}$

Fortunately, the first pilot programme supported by local government is being rolled out to clinics in each of the eight sub-districts in Cape Town during 2009. ${ }^{5}$ The City of Cape Town Council pledged their support to initiate communitybased infant hearing screening by partnering with a local non-governmental organisation, the Carel du Toit Centre Trust. Eight OAE machines were allocated for the purposes of screening the hearing of all infants attending their sixweek immunisation visit at eight primary health care clinics - a first for South Africa.

\section{Conclusion}

Equal opportunities for children with hearing loss are attainable and justifiable through effective early hearing detection and intervention programmes. Early intervention initiatives, such as the Carel du Toit Parent Guidance Programme and the HI HOPES (Home Intervention-Hearing and Language Opportunities Parent Education Services) home-based intervention programme are aimed at developing the potential of hearing-impaired children aged nought to three years. ${ }^{5}$

Collaborative efforts between the many shareholders in a hearing-impaired child's life are essential to increase awareness and advocacy at all levels. General practitioners and paediatricians are best placed to highlight and promote the importance of early identification to parents, hospital management and other health care providers. Furthermore, effective follow-up and referral systems are essential to ensure that those infants at risk of a hearing loss are tracked and provided with appropriate services within the critical period for language development. ${ }^{27}$

\section{Conflict of interest: None}

\section{References}

1. World Bank. World Development Report 2006 Equity and Development. New York: The World Bank and Oxford University Press; 2006.

2. World Health Organization. Preventing chronic diseases - a vital investment. Geneva; 2005.

3. Mehl A, Thomson V. Newborn hearing screening: The great omission. Pediatrics 1998;101(1):1-6.

4. Olusanya BO, Newton VE. Global burden of childhood hearing impairment and disease control priorities for developing countries. Lancet 2007;369:1314-7.

5. Swanepoel DW, Storbeck C, Friedland P. Early hearing detection and intervention in South Africa. Int $\mathrm{J}$ Pediatr Otorhinolaryngol 2009; International Journal of Pediatric Otorhinolaryngology, 73 (6), p.783-6, Jun 2009

6. Swanepoel D, Louw B, Hugo R. A novel service delivery model for infant hearing screening in South Africa. Int JAudiol 2007;46(6):321-7.

7. Yoshinaga-Itano C, Sedey AL, Coulter DK, Mehl AL. Language of early and later-identified children with hearing loss. Pediatrics 1998; 102:1161-71.

8. Moeller MP. Early intervention and language development in children who are deaf and hard of hearing. Pediatrics 2000;106(3):1-9.

9. Yoshinaga-Itano C. Levels of evidence: Universal newborn hearing screening (UNHS) and early hearing detection and intervention systems (EHDI). J Commun Disord 2004;37:451-65.

10. Kennedy C, McCann D, Campbell MJ, Kimm L, Thornton R. Universal newborn screening for permanent childhood hearing impairment: An 8-year follow up of a controlled trial. Lancet 2005;355:660-2.

11. Olusanya BO, Ruben RJ, Parving A. Reducing the burden of communication disorders in the developing world: An opportunity for the millennium development project. JAMA 2006;296:441-4

12. Mohr PE, Feldmann JJ, Dunbar JL, et al. The societal costs of severe to profound hearing loss in the United States. Int J Technol Assess Health Care 2000;16:1120-35.

13. Bieber FR, Nance WE. Hereditary hearing loss. In: Jackson LG, Schimke RN, eds. Clinical genetics: A source book for physicians. New York: Wiley; 1978:443-9

14. Cohn ES, Kelley PM, Fowler TW, et al. Clinical studies of families with hearing loss attributable to mutations in the connexin 26 gene GJB2/DFNB1). Pediatrics Mar 1999;103(3):546-50.

15. Arnos Kathleen. Ethical and social implications of genetic testing for communication disorders. J Commun Disord Sept/Oct 2008;41:444-57.

16. Professional Board for Speech, Language and Hearing Professions. Early Hearing Detection and Intervention Programmes in South Africa Position Statement. Pretoria: Health Professions Council of South Africa; 2007.

17. Bluestone CD. Pathogenesis of otitis media: Role of eustachian tube. Pediatr Infect Dis J Apr 1996;15(4):281-91.

18. Bluestone $C D$. Clinical course, complications and sequelae of acute otitis media. Pediatr Infect Dis J May 2000;19 S37-S46

19. Gravel JS, Roberts JE. Early otitis media with effusion, hearing loss, and auditory processes at school age. Ear Hear 2006;27:353-68.

20. Merifield DO, Parker NJ, Nicholson NC. Therapeutic management of chronic suppurative otitis media with otic drops. Otolaryngol Head Neck Surg. Jul 1993;109(1):77-82.

21. Lo PS, Tong MC, Wong EM, Van Hasselt CA. Parental suspicion of hearing loss in children with otitis media with effusion. Eur J Pediatr 2006 Dec;165(12):851-7.

22. Northern JL, Downs MP. Hearing in children. 5th ed. Baltimore, MA: Lippincott Williams \& Wilkens; 2002.

23. Olusanya BO, Swanepoel D, Chapman MJ, Castillo S, Habib H. Progress towards early detection services for infants with hearing loss in developing countries. BMC Health Services Research 2007, 7:14 Available at http://www.biomedcentral.com/1472-6963/7/14 (Accessed 27/10/09).

24. The Department of Health. The Primary Health Care Package for South Africa - a set of norms and standards. Available from http:// www.doh.gov.za/search/index.html (Accessed 10/04/2009).

25. Theunissen M, Swanepoel D. Early hearing detection and intervention services in the public health sector of South Africa. Int J Audiol 2008;47:S23-9.

26. The Department of Health. White Paper on the Transformation of the Health System in South Africa. Available from http://www.doh. gov.za/search/index.html (Accessed 10/04/2009).

27. Swanepoel D. Early detection of infant hearing loss in South Africa. SAMJ 2009 March; 99(3):158-9. 